Tuberkulose 1947;4(suppl 2):I-II

\title{
Inhaltsverzeichnis, Vol. 4, Supplementum 1, 1947
}

1. Teil: Die Tuberkulose-Morbidität des Kantons Zurich im Jahre 1945 von P. PRESS

I. Einleitung 1

II. Allgemeines 5

A. Allgemeine Einleitung

B. Tuberkulose-Morbidität im Kanton Zurich

C. Mortalität und Morbidität 9

III. Über die allgemeine Entwicklung der Tuberkulose 10

A. Erste Tuberkulose-Manifestation 10

Extrathorakale Tbc 10

Primär-Tbc 11

Sekundär-Tbc 12

Tertiäre Lungentbc

Offene Lungentbc

Geschlossene Lungentbc 15

5. Erythema nodosum

B. Evolution 19

Krankheitsentwicklung nach Primo-Infektion

Entwicklung der Tbc. nach Pleuritis

Allgemeine Entwicklung $\quad 22$

Lungentbc. nach Pleuritis 23

3. Krankheitsentwicklung der tertiären Lungentbc

Vorgängige tuberkulose, nicht tertiäre Manifestationen ... 26

Vorgängige tertiäre Schübe

27

Chirurgische Eingriffe. aa) Häufigkeit der KoUapstherapie bb) Die Bedeutung der verschiedenen Behandlungsmethoden

cc) Rückfälle nach erfolgter chirurgischer Behandlung . . 29

C. Besondere klinische Probleme

33

1. Die extrathorakale Tbc

33

Aufteilung nach Lokalisationen

Gleichzeitiges Vorkommen von Lungentbc. und extra-thora-kaler Tbc

2. Über Beginn- und Frühdiagnose der Lungentbc

Klinischer Beginn der Tbc

39

Zufällig entdeckte Lungentbc

40

Negative Resultate anläßl. vorgängiger Untersuchungen ... 41

Gleichzeitig bestehende, nicht tbc. Affektionen 
A. Aufteilung nach Geschlecht und Alter

B. Krankheitsdauer

48

Dauer des jetzigen Tbc.-Schubes

48

Frühere Schübe

50

Totale Krankheitsdauer $\quad 51$

C. KoUapstherapie 53

D. Aufenthaltsort 59

E. Die chronisch unheilbaren Offentuberkulösen

Tuberkulose Vol. IV, Supplementum II (1947) 1

2. Teíl: Bie Betreuuug der Tuberkulösen iin Kanton Zurich von M. BRUNNER und Mitarbeitern
I. Eî́nleitung
67
II. Organisation
68

A. Kantonale Gesundheitsdirektion

B. Stiftung Zürcherische Heilstätten Wald und Clavadel

C. Zürcher kantonale Liga gegen die Tuberkulose

68

Hire Entwickhmg und Aufgaben

71

Finanzielles 73

III. Zur Frage der Verstaatlíchung der Zürcher Heilstätten und der Arbeitsheilstätte Appisberg 76
A. Medizinischer Standpunkt
76
B. Wirtschaftlicher Standpunkt
77
C. Allgemeine Erwägungen
79
IV. Schirmbíld-Organisation
82

A. Vorbereitungen, die vor der röntgenologischen Untersuchung der Bevölkerung durchgeführt werden müssen

83

B. Photographische Aufhahme des Schirmbildes und Beurteilung desselben durch den Arzt 84

C. Abklärung der nach dem Schirmbild als krankhaft oder als zweifelhaft beurteilten Befunde der Thoraxorgane 84

D. Führung einer Kontrollkartei, anhand welcher die Abklärung überwacht werden kann 85

V. Die medizinische und sozílale Betreuung der Tuberkulösen

$\begin{array}{ll}\text { A. Ambulante Betreuung } & 86 \\ \text { Durch die praktizierenden Ärzte } & \\ \text { Durch die Polikliniken } & 87 \\ \text { Durch die Fürsorgesiellen } & 87\end{array}$

Tbc.-Fürsorgestellen der Stadt Zurich

Tbc.-Fürsorgestellen der Landschaft

86

87

B. Betreuung der Tuberkulösen durch Unterbringung in Anstalten

Betten-Kataster 95

Beziehungen zwischen den Anstalten und den Tbc.-Kranken

98

Präventorien

100

Erholungsheime 
Sanatorien $\quad 102$

Spitalstationen 105

Arbeitsheilstätte Appisberg

108

VI. Die aus der Arbeit hervorgegangenen drínglíchen Aufgaben

Ill

A. Schaffung einer permanenten Planungskommission Ill

B. Schaffung einer Kurnachweisstelle Ill

C. Unterbringung der chronisch Tuberkulösen 114

D. Schaffung einer Abteilung für geisteskranke Tuberkulose ...121

VII. Zusammenfassung

122

ABKÜRZUNGEN

$\mathrm{M}=$ Manner $\mathrm{F}=$ Frauen $\mathrm{K}=$ Kinder Tbc. $=$ Tuberkulose

tbc. $=$ tuberkulös $\quad$ EN $=$ Erythema nodosum $\quad$ Pn $=$ Pneumothorax

J. = Jahre 